\title{
Raising the Ras inhibitor bar
}

\section{By Michael J. Haas, Senior Writer}

About a year after U.S. researchers developed small molecule inhibitors of the intractable target wild-type Ras, a Japanese team has identified compounds that hit Ras mutant tumors in mice. ${ }^{1}$ The team now is trying to ramp up the potency of the mutant-targeting compounds and optimize the structures to make them more drug-like.

Ras family proteins play central roles in cell growth and proliferation. The proteins cycle between an inactivated, GDP-bound state (Ras-GDP) and an activated, GTP-bound state (Ras-GTP). Guanine nucleotide exchange factors such as son of sevenless homolog 1 (SOS1) facilitate Ras activation by promoting the release of GDP from inactivated Ras-thereby allowing intracellular GTP to bind and reactivate itand by enhancing the activation of Ras after it binds GTP.

\section{"Everyone is working with} what they've discovered. We're just coming at the goal from different-if somewhat overlapping - angles."

- Martin Drysdale,

The Beatson Institute for Cancer Research
Of the three Ras isoforms, K-Ras is most frequently dysregulated in cancer, with activating mutations found in about $80 \%$ of pancreatic cancers, $40 \%$ of colon cancers and $25 \%$ of lung cancers. Collectively, mutations in all three isoforms-K-Ras, v-Ha-ras Harvey rat sarcoma viral oncogene homolog (HRAS) and neuroblastoma Ras viral oncogene (NRAS) - occur in about $20 \%$ of all cancers.

Nevertheless, Ras proteins had long been considered undruggable because they lack well-defined surface pockets suitable for binding drug molecules. In 2012, independent groups at Vanderbilt University School of Medicine and the Genentech Inc. unit of Roche used fragment-based drug discovery to identify small molecules that blocked SOS1-mediated activation of wild-type K-Ras with mid-micromolar to high micromolar $\mathrm{IC}_{50}$ values. ${ }^{2-4}$

Meanwhile, a Japanese team led by Tohru Kataoka had been solving the cocrystal structures of wild-type and mutant Ras proteins bound to a GTP analog. Those studies revealed a previously unreported conformation of Ras-GTP that contained potentially druggable surface pockets. ${ }^{5-7}$

In the new study, Kataoka's team sought to identify small molecule inhibitors of the Ras-GTP conformation.

First, the group used structure-based computational models to screen a virtual library of about 41,000 molecules for compounds that blocked interactions between Ras-GTP and one of its downstream binding partners, CRAF (RAF1). The screen yielded 97 hits, and in vitro testing showed that one hit-Kobe0065-had low micromolar inhibitory activity against Ras-GTP.

Next, the team conducted a computer-based search of about 160,000 compounds and identified 273 that were structurally similar to Kobe0065. One of these-Kobe2602-also had micromolar activity against Ras-GTP in vitro.

In human colon, pancreatic, bladder and other cancer cell lines that harbored activating Ras mutations, Kobe0065 and Kobe2602 decreased growth compared with vehicle. Both compounds reduced growth in mutant Ras cell lines more effectively than they did in cancer cell lines expressing wild-type Ras.

In mice with xenograft tumors that harbored activating K-Ras mutations, two compounds lowered tumor growth compared with vehicle.

Additional in vitro studies showed that the compounds inhibited the interactions between activated Ras and its downstream targets such as Raf proteins, MEK and phosphoinositide 3-kinase (PI3K). The compounds also blocked binding between Ras-GTP and the allosteric domain of SOS1 that accelerates Ras-GTP activation.

Kataoka is professor of molecular biology and dean of the Kobe University Graduate School of Medicine. The team included researchers from the RIKEN SPring-8 Center, the Japan Synchrotron Radiation Research Institute and NEC Corp., which performed the computational screens and structure similarity searches.

Data were reported in the Proceedings of the National Academy of Sciences.

"Because the compounds work according to the desired mechanism of action and show in vitro and in vivo efficacy, this study moves the field forward and raises the bar in terms of what researchers will be able to publish on the Ras space in a good journal," Martin Drysdale told SciBX. "We're looking at this study to see if we can learn anything that applies to our own work" in targeting K-Ras with fragment-based approaches.

Drysdale is professor and head of the Drug Discovery Programme at The Beatson Institute for Cancer Research. Previously he was deputy research director at fragment-based and structure-based drug discovery company Vernalis plc.

\section{Convergence, not competition}

Kataoka said the effect of his team's compounds on downstream targets and the allosteric site of SOS1 made them clearly superior to the compounds reported by the Genentech and Vanderbilt groups. Those molecules inhibited Ras activation by blocking binding between RasGDP and the catalytic domain of SOS1.

Furthermore, he said, "it is doubtful whether those previously reported compounds have activity in mutant K-Ras-driven cancers because we showed in our study that inhibition of SOS1 did not alter the levels of Ras-GTP that carries activating mutations."

Drysdale agreed that blocking Ras-GTP was preferable to blocking Ras-GDP. 
"Conventional wisdom has it that something which blocks activated Ras-and most relevantly, mutated Ras-is the most obvious and sensible thing to do," he said. "The fact that the Kataoka team's compounds do that and affect molecules downstream of Ras is interesting."

However, he thinks it is too soon to conclude that the compounds from Kataoka's team are superior to those from the Genentech and Vanderbilt groups.

He said researchers who are developing Ras inhibitors-including his own group at Beatson-are working toward the same ultimate goal: inhibition of activated mutant Ras, which necessarily means inhibition of Ras-GTP.

"Everyone is working with what they've discovered," he said. "We're just coming at the goal from different-if somewhat overlapping-angles."

Indeed, Drysdale said the binding site of the Kataoka team's compounds overlaps with the site targeted by the Genentech and Vanderbilt inhibitors. "So to say that those earlier compounds bind only to Ras-GDP is an oversimplification," he said.

Drysdale also noted that the Kataoka team's compounds were far from being drug-like in terms of their potency and structure. "Even the authors point this out," he said. "Most people would not have tested these compounds at all. They are not even really good tool compounds."

Thus, he said, the compounds identified by the Kataoka, Genentech and Vanderbilt groups are all springboards for developing future inhibitors of activated, mutant Ras.

Kataoka's team is now conducting SAR studies on Kobe0065 and Kobe2602 to improve their potency and optimize their structures. In particular, the team wants to eliminate the compounds' thiosemicarbazide structure because "this is generally considered to lead to cellular toxicity," Kataoka said.

Kobe University has filed patent applications on the findings reported in PNAS, and the IP is available for licensing, he said.

Haas, M.J. SciBX 6(19); doi:10.1038/scibx.2013.454

Published online May 16, 2013

\section{REFERENCES}

1. Shima, F. et al. Proc. Natl. Acad. Sci. USA; published online April 29, 2013; doi:10.1073/pnas.1217730110

Contact: Tohru Kataoka, Kobe University Graduate School of Medicine, Kobe, Japan

e-mail: ataoka@people.kobe-u.ac.jp

Contact: Fumi Shima, same affiliation as above e-mail: sfumi@med.kobe-u.ac.jp

2. Sun, Q. et al. Angew. Chem. Int. Ed. 51, 6140-6143 (2012)

3. Maurer, T. et al. Proc. Natl. Acad. Sci. USA 109, 5299-5304 (2012)

4. Kotz, J. SciBX 5(21); doi:10.1038/scibx.2012.536

5. Ye, M. et al. J. Biol. Chem. 280, 31267-31275 (2005)

6. Shima, F. et al. J. Biol. Chem. 285, 22696-22705 (2010)

7. Muraoka, S. et al. FEBS Lett. 586, 1715-1718 (2012)

\section{COMPANIES AND INSTITUTIONS MENTIONED}

The Beatson Institute for Cancer Research, Glasgow, U.K. Genentech Inc., South San Francisco, Calif. Japan Synchrotron Radiation Research Institute, Hyogo, Japan Kobe University, Kobe, Japan

Kobe University Graduate School of Medicine, Kobe, Japan NEC Corp. (Tokyo:6701), Tokyo, Japan

RIKEN SPring-8 Center, Hyogo, Japan

Roche (SIX:ROG; OTCQX:RHHBY), Basel, Switzerland Vanderbilt University School of Medicine, Nashville, Tenn. Vernalis plc (LSE:VER), Winnersh, U.K. 